\title{
The DCD-N score could identify potential DCD donors
}

Successful donation of organs after cardiac death (DCD) is reliant on identification of patients who will die within 60 min of the withdrawal of life-sustaining treatment (WLST). "Potential DCD donors are missed in clinical practice in neurocritical care," explains Alejandro Rabinstein, from the Mayo Clinic, "owing to our inability to identify those patients who will die within 60 min of WLST". Now, Rabinstein et al. have validated a predictive score, termed the DCD-N score, aimed at successfully identifying this population of donors.

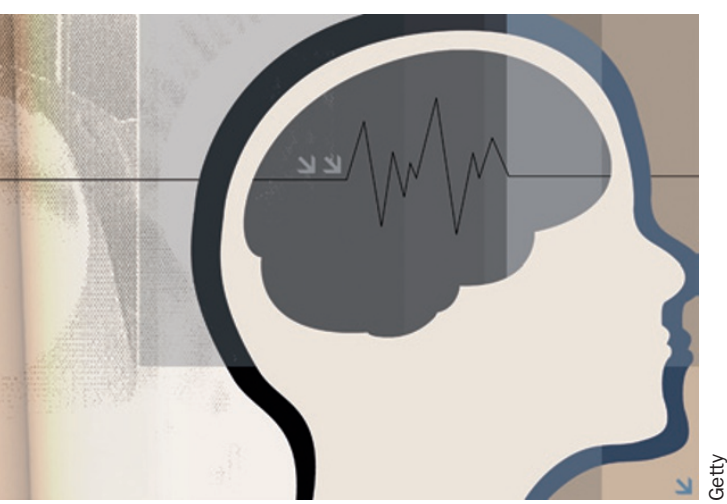

The researchers conducted a multicenter, observational study of 178 patients with irreversible brain injury undergoing WLST from six medical centers in the USA and The Netherlands. Four clinical characteristics-corneal reflex, cough reflex, best motor response and oxygenation index-were monitored in each patient. The primary end point of the study was death of the patient within $60 \mathrm{~min}$ of WLST. Regression analyses were conducted to assess the association of time of death with the predictor variables. Points were then attributed to each of the measured clinical characteristics to create the DCD-N score.

Of the 82 (46\%) patients who died within $60 \mathrm{~min}, 59$ had a DCD-N score of 3 or more (72\% sensitivity). Moreover, 75 of the 96 patients who did not die within $60 \mathrm{~min}$ had a DCD-N score of $0-2(78 \%$ specificity). Further analysis revealed that a score of 3 or more translated into a $74 \%$ chance of death within $60 \mathrm{~min}$ (that is the positive predictive value) whereas a score of 0-2 translated into a $77 \%$ chance of survival beyond $60 \mathrm{~min}$ (that is the negative predictive value).

"The main finding of this study is that four simple clinical variables, which are readily available at the bedside without removal of the patient from mechanical ventilation, can identify those patients who have good potential as DCD donors (that is those that will die within $60 \mathrm{~min}$ of WLST). Moreover, this score was shown to have good sensitivity and specificity", explains Rabinstein. This study therefore demonstrates the effectiveness of the DCD-N score in identifying good potential DCD donors from those patients with nonsurvivable brain injury. The researchers now plan to conduct further studies "to compare the DCD-N score with currently used scoring sytems."

Katherine Smith

Original article Rabinstein, A. A. et al. Prediction of potential for organ donation after cardiac death in patients in neurocritical state: a prospective obervational study. Lancet Neurol. 11, 414-419 (2012) 